\title{
Lexical selection in multi-word production
}

\author{
Niels Janssen ${ }^{1 *}$ and Alfonso Caramazza ${ }^{2,3}$ \\ 1 Psycholinguistics and Cognitive Neuroscience Group, Department of Psychology, University of La Laguna, Tenerife, Spain \\ 2 Department of Psychology, Harvard University, Cambridge, MA, USA \\ ${ }^{3}$ Centre for Mind/Brain Sciences, University of Trento, Trento, Italy
}

Edited by:

Sonja A. E. Kotz, Max Planck Institute

Leipzig, Germany

Reviewed by:

Judith F. Kroll, Penn State University, USA

Luciano Fadiga, University of Ferrara, Italy

\section{${ }^{*}$ Correspondence:}

Niels Janssen, Psycholinguistics and Cognitive Neuroscience Group,

Department of Psychology, University

of La Laguna, Campus of Guajara,

38205 Tenerife, Spain.

e-mail:njanssen@ull.es
In multi-word utterances, target words need to be selected in the context of other target words. In the present study, three hypotheses were tested that differed in their assumptions about whether the lexical selection mechanism considers the activation levels of the other target lexical representations, and whether it takes into account their grammatical class properties. Participants produced adjective + noun and noun + noun utterances in response to colored word and picture + word stimulus displays. In both types of utterances, the frequency of the first and second response was manipulated. The results revealed an effect of the frequency of the second response that did not depend on the utterance type, and additive effects for the frequency of the first and the second response in both utterance types. These results are interpreted in terms of a model of lexical selection that assumes that selection is non-competitive.

Keywords: language production, multi-word naming, noun-phrase production, competitive lexical selection, lexical frequency

\section{INTRODUCTION}

A hotly debated topic in the language production literature concerns the nature of the lexical selection mechanism. This debate has primarily focused on data from single-word production (e.g., Miozzo and Caramazza, 2003; Belke et al., 2005; Hantsch et al., 2005; Finkbeiner and Caramazza, 2006; Howard et al., 2006; Kuipers et al., 2006; Abdel Rahman and Melinger, 2007; Mahon et al., 2007; Dell et al., 2008). In single-word production, lexical selection takes place in the context of other lexical representations that are not targets. By contrast, in multi-word production, target lexical selection takes place in the context of other lexical representations that are also targets. In addition, target selection takes place in the context of other targets whose grammatical class properties either differ or do not differ from the target's grammatical class property. The question we addressed in the current paper is whether the lexical selection mechanism is influenced by the activation levels of the other target lexical representations, and whether it takes into account the grammatical class information of the to be produced words. In the experiment, participants produced adjective + noun and noun + noun utterances in which the frequency of the first and second response was manipulated.

There are three hypotheses in the literature that differ in their assumptions about whether lexical selection considers the activation levels of other lexical representations and whether it takes into account their grammatical class property. First, the Grammatically Unconstrained Competitive lexical selection hypothesis (i.e., Garrett, 1980; Vigliocco et al., 2004) assumes that target lexical selection times are a function of the activation levels of other lexical representations irrespective of their grammatical class property. Second, the Grammatically Constrained Competitive lexical selection hypothesis (i.e., Levelt et al., 1999; Pechmann and Zerbst, 2002; Dell et al., 2008) assumes that target lexical selection times are a function of the activation levels of other lexical representations, but only if they are from the same grammatical class as the target. Finally, the Non-Competitive lexical selection hypothesis (i.e., Dell, 1986) assumes that target lexical selection times are not influenced by the activation levels of other lexical representations, irrespective of their grammatical class property.

One way to distinguish between these three hypotheses would be to consider their predictions regarding the selection times of the first response as a function of the frequency of the second response in adjective + noun and noun + noun utterances. We illustrate the predictions of the three hypotheses by means of the top, middle, and bottom rows of Figure 1. On the assumption that frequency affects the activation level of a lexical representation (e.g., McClelland and Rumelhart, 1981)' , the Grammatically Unconstrained Competitive lexical selection hypothesis (top row) predicts that lexical selection times for the first response (leftmost column) should increase with increasing frequency of the second response in both adjective + noun and noun + noun utterances. According to this hypothesis, lexical selection is competitive and not constrained by grammatical class, and so the selection time for the lexical representation corresponding to the first response should increase with increasing levels of activation of the lexical representation corresponding to the second response, irrespective of whether the responses have the same or different grammatical class properties.

By contrast, the Grammatically Constrained Competitive lexical selection hypothesis (middle row) predicts that lexical selection times for the first response should increase with increasing

${ }^{1}$ Note that the model of Levelt et al. (1999) assumes that the frequency effect resides at a morpho-phonological level of representation. Importantly, this level is independent from the level at which lexical selection is assumed to take place. However, there exists a myriad of data that challenges a morpho-phonological locus of the frequency effect (e.g., Caramazza et al., 2001; Bonin and Fayoll, 2002; Navarrete et al., 2006; Janssen et al., 2008). As such it is safe to assume that word selection and word frequency affect the same level of processing. 


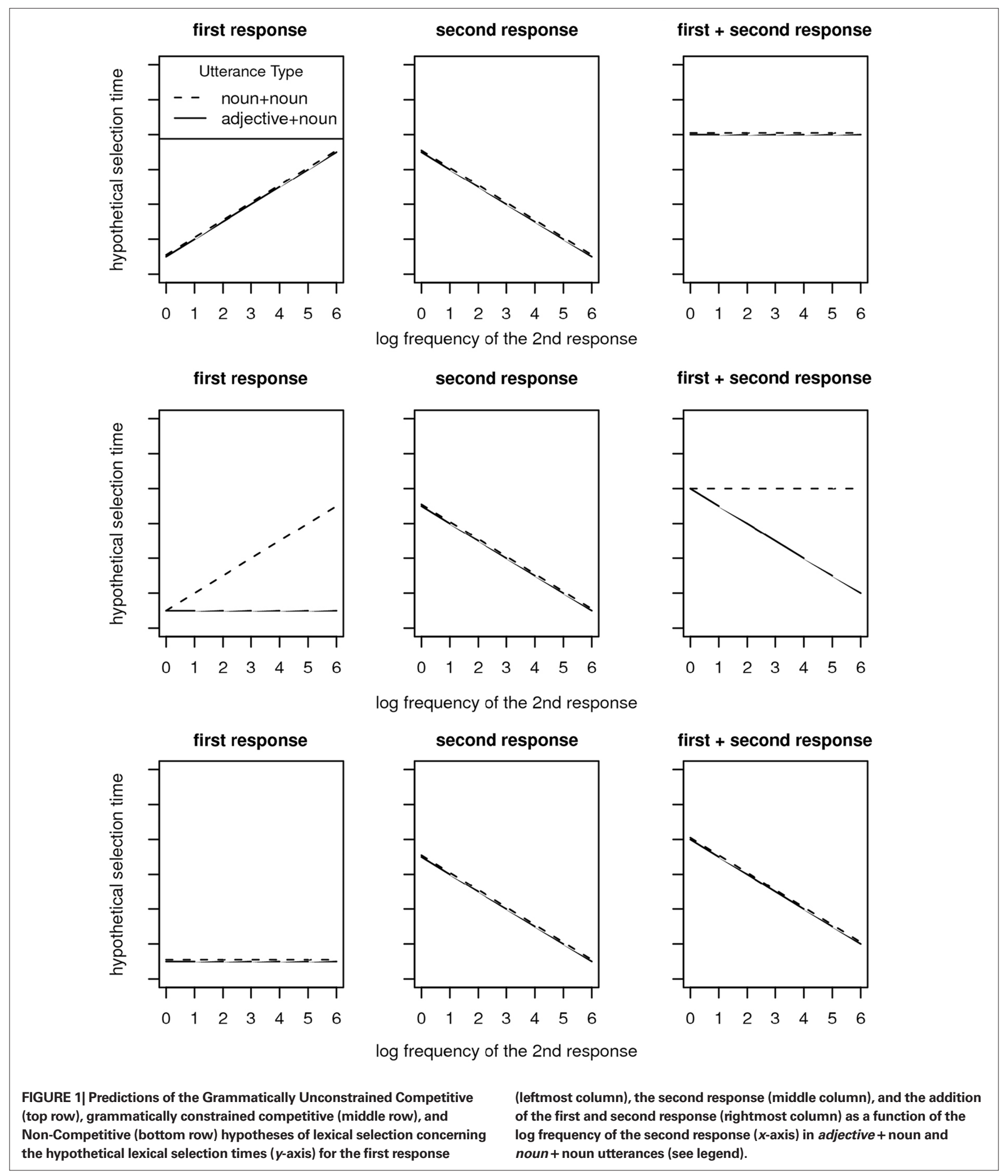

frequency of the second response in noun + noun, but not in adjective + noun utterances. This is because this hypothesis assumes that lexical selection is competitive and grammatically constrained, and so the selection time for the lexical representation corresponding to the first response should only be influenced by activation levels of the lexical representation corresponding to the second response, if the two responses have the same grammatical class properties. 
Finally, the Non-Competitive lexical selection hypothesis (bottom row), predicts that lexical selection times for the first response should not increase with increasing frequency of the second response in both adjective + noun and noun + noun utterances. This is because this hypothesis assumes that lexical selection is not competitive, and so the selection time for the lexical representation corresponding to the first response should be independent from the level of activation of the lexical representation corresponding to the second response, irrespective of whether the responses have the same or different grammatical class properties.

Further, with respect to the selection time for the second response in adjective + noun and noun + noun utterances (middle column), all three hypotheses predict that lexical selection time should decrease with increasing frequency. Consequently, with respect to the total lexical selection time (rightmost column), the Grammatically Unconstrained Competitive lexical selection hypothesis predicts that it should not vary as a function of the frequency of the second noun in adjective + noun and noun + noun utterances, the Grammatically Constrained Competitive hypothesis predicts that it should vary as a function of the frequency of the second noun in adjective + noun, but not in noun + noun utterances, and the Non-Competitive hypothesis predicts that it should vary as a function of the frequency of the second noun in both adjective + noun and noun + noun utterances.

Previous studies investigating multi-word production have not focused on the question of how word selection takes place in a multi-word context. Instead, these studies have focused on general issues related to the interplay between grammatical and phonological information in the production of multi-word utterances (Bock, 1987; Cleland and Pickering, 2003; Smith and Wheeldon, 2004; Janssen and Caramazza, 2009), and on more specific issues related to the planning and sequentiality of multi-word production (Schriefers et al., 1999; Alario and Caramazza, 2002; Smith and Wheeldon, 2004; Morgan and Meyer, 2005; Damian and Dumay, 2007; Malpass and Meyer, 2010; Wagner et al., 2010).

Regarding the relationship between grammatical and phonological processing, Janssen and Caramazza asked native English participants to produce noun + noun and adjective + noun utterances that either shared phonological onset (ring rake $_{\mathrm{N}}, \operatorname{red}_{\mathrm{A}}$ rake $_{N}$ ) or were unrelated ( gun $_{N}$ rake $_{N}$, green ${ }_{A}$ rake $_{N}$ ). To elicit the noun + noun utterances a picture + word stimulus display was used, and to elicit the adjective + noun utterances a colored word display was used. The results revealed that the phonological effect was modulated by the type of utterance: Phonological facilitation effects were found for adjective + noun utterances, and inhibition effects were found for noun + noun utterances. Subsequent experiments ruled out an influence of the stimulus display on these data, and generalized the results to other utterance types. On the basis of these data, Janssen and Caramazza argued in favor of models that assume that syntactic decisions have direct access to a word's phonological information (Alario and Caramazza, 2002).

Addressing the issue of planning and sequentiality, Alario et al. (2002) asked native English participants to name colored pictures with adjective + noun utterances (e.g., blue kite $_{\mathrm{N}}$ ). The frequency of the adjective and noun was manipulated factorially. The results revealed that naming latencies decreased with increasing frequency of the adjective and of the noun, and that these two frequency effects were additive. These results led Alario et al. to draw two conclusions about the planning and sequentiality of word retrieval in a multiword context. First, they argued that the main effects for adjective and noun frequency reveal that the scope of lexical processing encompasses both responses in the adjective + noun utterance (see also Meyer, 1996). Second, they interpreted the additive effects of adjective and noun frequency in terms of sequential lexical selection, where the total lexical selection time of the responses in an utterance is equal to the sum of the lexical selection times of each individual response in the utterance (but see Malpass and Meyer, 2010).

The data of Alario et al. (2002) can also be used to distinguish between the predictions of the Grammatically Unconstrained Competitive, Grammatically Constrained Competitive, and Non-Competitive hypotheses. In particular, they are inconsistent with the Grammatically Unconstrained Competitive lexical selection hypothesis, but consistent with the Grammatically Constrained Competitive and Non-Competitive hypotheses. Thus, the Grammatically Unconstrained Competitive lexical selection hypothesis predicts that naming latencies should not vary as a function of the frequency of the second noun in adjective + noun utterances. In contrast to this prediction, the results of Alario et al. revealed that naming latencies decreased with increasing frequency of the second noun. However, the results of Alario et al. do not distinguish between the Grammatically Constrained Competitive and Non-Competitive hypotheses. They both predict that naming latencies should decrease with increasing frequency of the second noun in adjective + noun utterances, a prediction that is in line with Alario et al.'s observations. As we argued above, the predictions of these three hypotheses can be further distinguished by comparing their predictions regarding a manipulation of the frequency of the second noun in adjective + noun and noun + noun utterances. We directly tested these predictions in the experiment below.

In addition, we examined the sequentiality of word retrieval by also varying the frequency of the first response in the two utterance types. Following Alario et al. (2002), if word retrieval if sequential, we would expect additive effects of the frequency of the first and second response in the two utterance types. The noun + noun and adjective + noun utterances were elicited by the same picture + word and colored word stimulus displays as used by Janssen and Caramazza (2009; see also Smith and Wheeldon, 2004).

\section{EXPERIMENT \\ METHODS \\ Participants}

Twenty native English speakers, undergraduate students from Harvard University, participated in the experiment. They were paid $\$ 5$ or received course credit upon completion of the experiment.

\section{Materials and design}

There were three variables in the experiment: The factor Type of Utterance with two levels (adjective + noun and noun + noun), the variable frequency of the first response, and the variable frequency of the second response. The adjective + noun stimuli consisted of a colored word, where the color name was the first response 
and the word the second response. The noun + noun stimuli were composed of a picture and a superimposed word, where the picture name was the first and the word the second response. The colors and objects to be used as the first response in these two naming conditions were selected to have similar properties. The names of the four colors were RED, GREEN, BLUE, and PINK, and the names of the four objects were RING, GLASS, BALL, and PIPE. The color and object names thus shared initial onset consonants, and had similar frequency estimates (color names: 132 [46-188], object names: 80 [23-132]).

A set of 80 concrete nouns was chosen to be used as the second response in the adjective + noun and noun + noun utterances (see Table A1 in Appendix). These words were all listed as nouns in the CELEX lexical database (Baayen et al., 1993) and had a higher noun than verb lexical frequency rating. The nouns varied in frequency across a wide range (1-394), and their imageability, and bigram frequency estimates were also collected. Imageability and Bigram Frequency were not correlated with frequency $(r=-0.02$ for frequency and imageability, and $r=-0.02$ for frequency and bigram frequency). For the purposes of constructing the experimental materials, the group of nouns was divided into two equal sets of low frequency and high frequency nouns. To form the adjective + noun and noun + noun conditions, each individual color and object name was paired with 10 low frequent and 10 high frequent nouns. There was no phonological, semantic, or associative relationship between the words in a pair. The pairing of colors and objects with words led to a total of 160 experimental pairs ( 80 for the color and 80 for the object naming condition). Four additional words to be used as a second response were selected that were paired with pictures and colors to form two sets of 16 practice items.

Each experimental item was assigned to one of five blocks such that each condition appeared equally within a given block. The trials in each block were pseudo-randomized such that on consecutive trials there was no semantic or phonological overlap between the first or second response across trials. The order of blocks was counterbalanced across participants using five distinct stimulus lists. Given that the color and object names shared initial onset consonants, the stimulus lists created for the adjective + noun condition were also used for the noun + noun condition. Finally, the order of Utterance Type was blocked, and was counterbalanced across participants.

\section{Procedure}

The experimental software was DMDX (Forster and Forster, 2003). The experiment consisted of two parts in which either noun + noun or adjective + noun stimuli were named. Participants either started with the noun + noun or with the adjective + noun part. Each part consisted of a practice phase and an experimental phase with the same trial structure. In the noun + noun part, participants were told that they would see one of four pictures on the screen with a superimposed word written inside. They were then told the names of the four pictures. They were told to name first the picture and then the superimposed word. In the adjective + noun part, participants were told they would see words presented in one of four colors on the screen. They were told to name first the color and then the superimposed word. On each trial, first a fixation point appeared for $700 \mathrm{~ms}$ and was replaced by a blank screen for $200 \mathrm{~ms}$. Next, the stimulus appeared on the screen and remained on the screen for $1500 \mathrm{~ms}$ independently of whether the voicekey triggered. Next, a blank screen was presented for $2000 \mathrm{~ms}$ before the next trial started.

The duration of the experiment was approximately $25 \mathrm{~min}$.

\section{ANALYSES AND RESULTS}

Trials on which the participant produced an incorrect response, hesitated, or made any other non-vocal sound were discarded, as well as trials on which the RT was below 300 ms (51 trials out of 3200, or $1.6 \%)$. In addition, outliers were discarded based on visual analysis of the quantile-quantile plots (34 trials, $1.1 \%$ ). The resulting set of 3115 data points was analyzed using a linear mixed effects methodology (Bates, 2005; Baayen, 2008). The raw naming latency data were inverse transformed in an attempt to reduce skewness and more closely approach a normal distribution. These transformed RTs were then fitted to a linear mixed model with participants and items as random effects, and utterance type, frequency of the first response, frequency of the second response, and their interactions as fixed effects. The frequency variables were log transformed to reduce skewness.

A graphical representation of the effect of the frequency of the second response in the two utterance types is presented in Figure 2. The variables that produced significant effects on naming latencies are presented in Table 1. Table 1 reveals that naming latencies were sensitive to the frequency of the first response, and to the frequency of the second response. There was no main effect of utterance type $(t<1)$, nor were there double interactions between utterance type and frequency of the first response $(t(3108)=1.52, p=0.13)$, between utterance type and the frequency of the second response $(t<1)$, and between the frequency of the first and frequency of the second response $(t<1)$. The triple interaction between utterance type, frequency of the first response, and frequency of second response was not significant $(t<1)^{2}$.

In other words, RTs decreased with increasing values of the frequency of the second response in the same way for noun + noun and adjective + noun utterances, as indicated by significant effects of the frequency of the second response, and by the absence of the interaction between utterance type and the frequency of the second response. In addition, the effects of the frequency of the first response and the frequency of the second response were additive, given that there were main effects of these two variables, and no interaction. Error analyses using logistic regression did not yield any significant results.

\section{GENERAL DISCUSSION}

The results reported here revealed that in the production of adjective + noun and noun + noun utterances, naming latencies decreased with increasing frequency of the second noun in the same manner for both utterance types. In addition, there were main effects for frequency of the first and second response, and there was no interaction between them (see also Alario et al., 2002).

These results are inconsistent with the predictions of the Grammatically Unconstrained Competitive and Grammatically Constrained Competitive hypotheses of lexical selection. The Grammatically Unconstrained Competitive lexical selection

${ }^{2}$ The variable phrase frequency (e.g., Arnon and Snider, 2010) predicted latencies, but did not interact with the other frequency variables. 


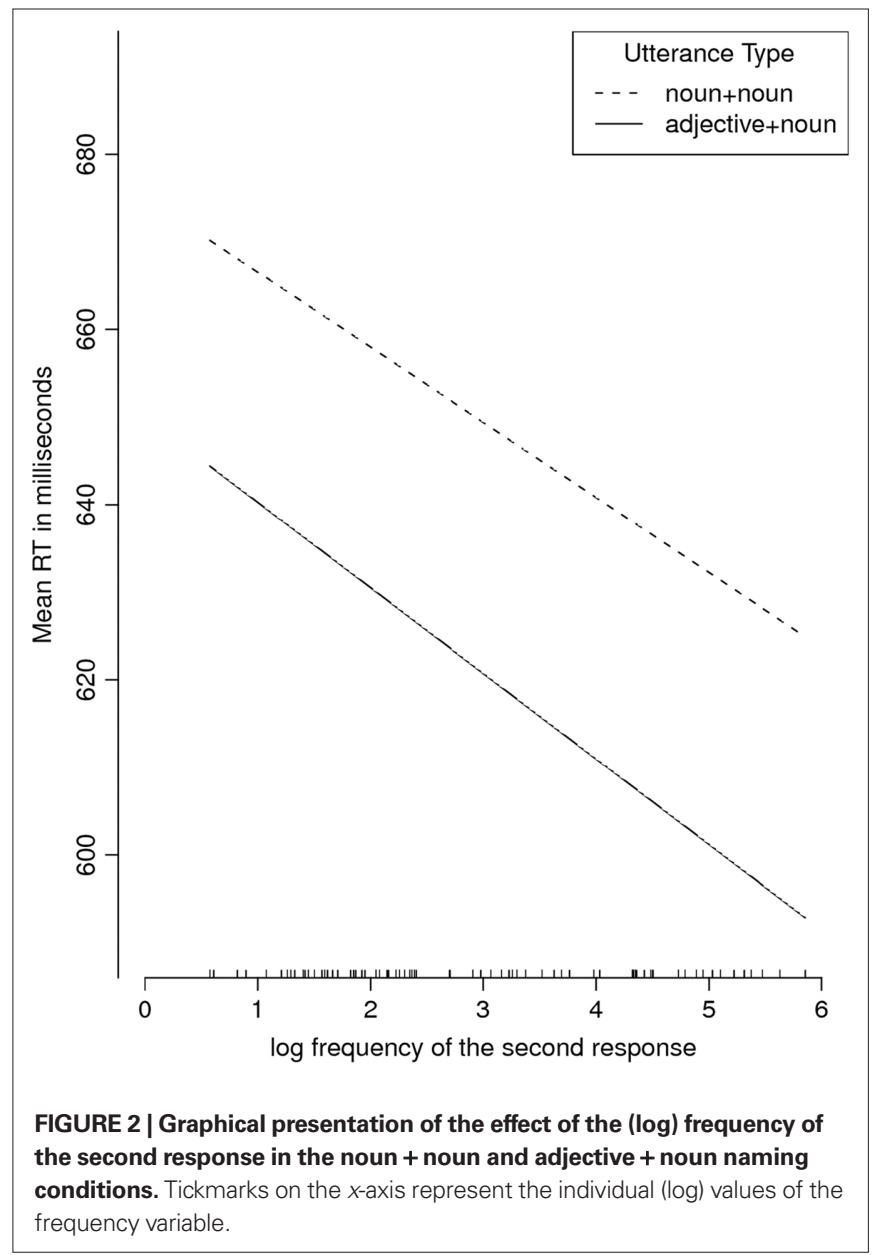

Table 1 | Beta (standard error), $\boldsymbol{t}$ (degrees of freedom), and $\boldsymbol{p}$-vales for the fixed effects in the linear mixed effect analysis.

\begin{tabular}{llrl}
\hline Variables & $\boldsymbol{\beta}$ (SD) & $\boldsymbol{T ( 3 1 0 8 )}$ & $\boldsymbol{p}$ (mcmc) \\
\hline (Intercept) & $-1.52 \mathrm{E}-03(5.57 \mathrm{E}-05)$ & -27.20 & 0.00 \\
Freq first response & $-3.08 \mathrm{E}-05(1.32 \mathrm{E}-05)$ & -2.33 & 0.02 \\
Freq second response & $-5.94 \mathrm{E}-07(2.91 \mathrm{E}-07)$ & -2.04 & 0.03 \\
\hline
\end{tabular}

p Values were calculated by Markov chain Monte Carlo confidence intervals sampled from the posterior distribution of the independent variables using the "language R" package in $R$ (Baayen, 2008).

hypothesis (e.g., Vigliocco et al., 2004) predicts that naming latencies should not vary as a function of the frequency of the second noun in adjective + noun and noun + noun utterances. The Grammatically Constrained Competitive hypothesis (e.g., Levelt et al., 1999; Pechmann and Zerbst, 2002; Dell et al., 2008), predicts that naming latencies should decrease with increasing frequency of the second noun in adjective + noun, but not in noun + noun utterances. In contrast to these predictions, naming latencies decreased with increasing frequency of the second noun in both adjective + noun and noun + noun utterances. These data are consistent with the predictions of the Non-Competitive hypothesis of lexical selection, that assumes that lexical selection does not take into account the activation of other lexical items, and predicts that naming latencies should decrease with increasing frequency of the second noun (see Figure 1).

This interpretation of the results relies on the background assumption that the selection of the responses in the utterances takes place in a sequential fashion (Alario et al., 2002). One might object to this assumption and propose that lexical selection is competitive and that it occurs in parallel (Schriefers et al., 1999). If such were the case, one would expect that the total selection time of the responses in an utterance to be a function of the response with the longest selection time. To explain the results reported here, one could propose that the selection time for the second response is slower than the selection time for the first response. Under such circumstances, the total selection time would not be a function of the selection time of the first response, and would therefore not reflect any potential competition effects. However, this explanation of the results is unlikely. If lexical selection were to occur in parallel and total selection time were not to reflect selection of the first response, one would expect no effect of the frequency of the first response on naming latencies. In contrast to this prediction, the current data, and those reported by Alario et al. (2002) revealed a clear effect of the frequency of the first response.

A further objection might be that the competition effect associated with the selection of the first response could be much smaller than the frequency effect associated with the selection of the second response. If such were the case, one would expect naming latencies to vary only with the frequency of the second noun in the adjective + noun and noun + noun utterances. However, this explanation leads to the incorrect prediction that the competition effect should be revealed if participants were to produce only the first response (i.e., the picture or color) and not the second response (i.e., the word) upon viewing the picture + word and colored word stimuli used here. In contrast to this prediction, Miozzo and Caramazza (2003) demonstrated that picture naming latencies were faster in the context of high versus low frequency distracter words - the opposite from what is expected under the assumption of competitive lexical selection. Thus, the possibility that the competition effect was overshadowed by the frequency effect also seems like an unlikely explanation of the results observed here.

A potential concern is that the picture-word and colored word tasks used here do not tap into normal language production processes, and hence, one cannot draw strong conclusions from the results presented here. However, there are at least three reasons that mitigate this concern. First, in both picture-word and colored word tasks, naming latencies were sensitive to the lexical frequency of the first and second response. Lexical frequency effects are generally taken as signature effects of lexical access (Almeida et al., 2007), implying that normal production processes occurred. Second, our results obtained with the colored word task replicate those obtained by Alario et al. (2002). Given that Alario et al's study employed colored pictures, these results generalize across different experimental paradigms, suggesting that they reflect more general aspects of language production. Finally, the two experimental tasks used in the current study have recently been used to examine other aspects of language production. As discussed in the Introduction, Janssen and Caramazza (2009) have reported contrasting effects of phonological onset similarity between utterances elicited by various stimulus displays including the ones 
tested here. Importantly, the same results were found in different stimulus displays, and different results were found within a single display, suggesting that it is not stimulus display but general language production processes that account for the observed pattern of results. ${ }^{3}$ Thus, on the basis of these arguments, one can safely assume that the two tasks used here tap into normal language production processes.

Studies in single-word production have also looked at the question of whether lexical selection considers the activation levels and grammatical class of other non-target lexical representations. Among the evidence that has been brought to bear on this question is the semantic interference effect in the picture-word interference paradigm (e.g., Hantsch et al., 2005; Kuipers et al., 2006), and the semantic blocking and cumulative semantic effects in the bare picture naming paradigm (e.g., Kroll and Stewart, 1994; Belke et al., 2005; Damian and Als, 2005; Howard et al., 2006; Abdel Rahman and Melinger, 2007) ${ }^{4}$. For example, Kroll and Stewart (1994) observed that naming latencies to pictures were slower when those pictures were presented in a semantically related compared to an unrelated context, a result that was interpreted in terms of competitive lexical selection. However, it should be clear that only to the extent to which these effects arise at a lexical level can they be used as evidence in favor of a competitive lexical selection hypothesis. With respect to the semantic interference effect, recent evidence is incompatible with a lexical locus of this effect (Miozzo and Caramazza, 2003; Finkbeiner and Caramazza, 2006; Kuipers

${ }^{3}$ This study also ruled out a role for the separability of the two stimulus elements in the displays, as well as the high number of repetitions of the first response in the adjective + noun and noun + noun utterances.

${ }^{4}$ Besides these behavioral data, evidence cited in favor of competition comes from domains using electrophysiological (Aristei et al., 2011), and hemodynamic measures (e.g., Snyder et al., 2010).

\section{REFERENCES}

Abdel Rahman, R., and Melinger,A. (2007). When bees hamper the production of honey: lexical interference from associates in speech production. J. Exp. Psychol. Learn. Mem. Cognit. 33, 604-614.

Alario, F.-X., and Caramazza, A. (2002). The production of determiners: evidence from French. Cognition 82, 179-223.

Alario, F.-X., Costa, A., and Carmazza, A. (2002). Frequency effects in noun phrase production: implications for models of lexical access. Lang. Cogn. Process. 17, 299-319.

Almeida, J., Knobel, M., Finkbeiner, M., and Caramazza, A. (2007). The locus of the frequency effect in picture naming: When recognizing is not enough? Psychon. Bull. Rev. 14, 1177-1182.

Aristei,S., Melinger,A., and Abdel Rahman, R. (2011). Electrophysiological chronometry of semantic context effects in language production. J. Cogn. Neurosci. 23, 1567-1586.

Arnon, I., and Snider, N. (2010). More than words: frequency effects for multi-word phrases. J. Mem. Lang. $62,67-82$.

Baayen, R. H. (2008). Analyzing Linguistic Data. A Practical Introduction to Statistics Using $R$. Cambridge: Cambridge University Press.

Baayen, R. H., Piepenbrock, R., and van Rijn, A. (1993). The CELEX Lexical Database [CD-ROM]. Philadelphia: Linguistic Data Consortium, University of Pennsylvania.

Bates, D. M. (2005). Fitting linear mixed models in R. R News 5, 27-30.

Belke, E., Meyer, A. S., and Damian, M. F. (2005). Refractory effects in picture naming as assessed in a semantic blocking paradigm. Q. J. Exp. Psychol. A Hum. Exp. Psychol. 58A, 667-692.

Bock, K. (1987). An effect of the accessibility of word forms on sentence structures. J. Mem. Lang. 26, 119-137.

Bonin, P., and Fayoll, M. (2002). Frequency effects in the written and spoken production of homophonic picture names. Eur. J. Cognit. Psychol. 14, 289-313.

Caramazza, A., Miozzo, M., Costa, A., and Bi, Y. (2001). The whole-word

et al., 2006; Mahon et al., 2007). And with respect to the semantic blocking and cumulative semantic effect, an exact locus of the effect has yet to be determined, but it has been suggested that at least part of this effect arises in learning or memory stages (e.g., Damian and Als, 2005; Navarrete et al., 2010).

One interpretation of the results from the single-word production studies is that the mechanism of lexical selection is not competitive: Lexical selection time is not influenced by the activation levels of other non-target lexical representations (Mahon et al., 2007). The present study reveals evidence from multi-word production that converges with this interpretation. The results reported here suggests that the time it takes to select a lexical representation is not affected by the activation levels of other target lexical representations - even if they are from the same grammatical class as the target. These results find a clear explanation in terms of the Non-Competitive hypothesis that assumes that the mechanism of lexical selection considers the grammatical class property of the other words in the utterance, and only the activation level of the target (e.g., Dell, 1986). This hypothesis predicts that target selection times would decrease with increasing activation, independently of the level of activation of other lexical representations. In turn, this would predict that naming latencies should decrease with increasing frequency of the second noun in both adjective + noun and noun + noun utterances, which is precisely the pattern observed here.

\section{ACKNOWLEDGMENTS}

The research reported here was supported by a Juan de la Cierva post-doctoral fellowship from the Spanish Ministry of Science and Technology to Niels Janssen, and by Grant R01DC006842 from the National Institute on Deafness and Other Communication Disorders, and the Società Mente/Cervello, Fondazione Cassa di Risparmio di Trento e Rovereto to Alfonso Caramazza.

frequency effect: implications for the representations of homophones. $J$. Exp. Psychol. Learn. Mem. Cogn. 27, 1430-1450.

Cleland, A. A., and Pickering, M. J. (2003). The use of lexical and syntactic information in language production: evidence from the priming of nounphrase structure. J. Mem. Lang. 49, 214-230.

Damian, M. F., and Als, L. C. (2005). Longlasting semantic context effects in the spoken production of object names. $J$. Exp. Psychol. Learn. Mem. Cognit. 31, 1372-1384.

Damian, M. F., and Dumay, N. (2007) Time pressure and phonological advance planning in spoken production. J. Mem. Lang. 57, 195-209.

Dell, G. S. (1986). A spreading-activation theory of retrieval in sentence production. Psychol. Rev. 93, 283-321.

Dell, G. S., Oppenheim, G. M., and Kittredge, A. (2008). Saying the right word at the right time: syntagmatic and paradigmatic interference in sentence production. Lang. Cogn. Process. 23, 583-608.
Finkbeiner, M., and Caramazza,A. (2006). Now you see it, now you don't: on turning semantic interference into facilitation in a Stroop-like task. Cortex 42, 790-796.

Forster, K. I., and Forster, J. C. (2003). DMDX: a windows display program with millisecond accuracy. Behav. Res. Meth. Instrum. Comput. 35, 116-124.

Garrett, M.F. (1980). "Levels of processing in sentence production," in Language Production, Vol. 1, Speech and Talk, ed. B. Butterworth (New York: Academic Press), 133-177.

Hantsch, A., Jescheniak, J. D., and Schriefers, H. (2005). Semantic competition between hierarchically related words during speech planning. Mem. Cognit. 33, 984-1000.

Howard, D., Nickels, L., Coltheart, M. and Cole-Virtue, J. (2006). Cumulative semantic inhibition in picture naming: experimental and computational studies. Cognition 100, 464-482.

Janssen, N., Bi, Y., and Caramazza, A. (2008). A tale of two frequencies: determining the speed of lexical access 
for Mandarin Chinese and English compounds. Lang. Cogn. Process. 23, 1191-1223.

Janssen, N., and Caramazza, A. (2009). Grammatical and phonological influences on word order. Psychol. Sci. 20, 1262-1268.

Kroll, J. F., and Stewart, E. (1994). Category interference in translation and picture naming: evidence for asymmetric connections between bilingual memory representations. J. Mem. Lang. 33, 149-174.

Kuipers, J.-R., La Heij, W., and Costa, A. (2006).A further look at semantic contexts effects in language production: the role of response congruency. Lang. Cogn. Process. 21, 892-919.

Levelt, W. J. M., Roelofs, A., and Meyer, A. S. (1999). A theory of lexical access in speech production. Behav. Brain Sci. 22, 1-75.

Mahon, B.Z., Costa,A., Peterson, R., Vargas, K.A., and Caramazza,A. (2007). Lexical selection is not by competition: a reinterpretation of semantic interference and facilitation effects in the picture-word interference paradigm. J. Exp. Psychol. Learn. Mem. Cognit. 33, 503-535.

Malpass, D., and Meyer, A. S. (2010). The time course of name retrieval during multiple-object naming: evidence for extrafoveal-on-foveal effects. $J$. Exp. Psychol. Learn. Mem. Cognit. 36, 523-537.

McClelland, J. L., and Rumelhart, D. E. (1981). An interactive activation model of context effects in letter perception. Psychol. Rev. 88, 375-407.

Meyer, A. S. (1996). Lexical access in phrase and sentence production: results from picture-word interference experiments. J. Mem. Lang. 35, 477-496.

Miozzo, M., and Caramazza, A. (2003). When more is less: a counterintuitive effect of distractor frequency in the picture-word interference paradigm. J. Exp. Psychol. Gen. 132, 228-252.

Morgan, J. L., and Meyer, A. S. (2005). Processing of extrafoveal objects during multiple-object naming. J. Exp. Psychol. Learn. Mem. Cognit. 31, 428-442.

Navarrete, E., Basagni, B., Alario, F.-X., and Costa, A. (2006). Does word frequency affect lexical selection in speech production? Q. J. Exp. Psychol. 59, 1681-1690.

Navarrete, E., Mahon, B. Z., and Caramazza,A. (2010). The cumulative semantic cost does not reflect lexical selection by competition. Acta Psychol. 34, 279-289.

Pechmann, T., and Zerbst, D. (2002). The activation of word class information during speech production. J. Exp. Psychol. Learn. Mem. Cognit. 28 233-243.

Schriefers, H., de Ruiter, J. P., and Steigerwald, M. (1999). Parallelism in the production of noun phrases: experiments and reaction time models. J. Exp. Psychol. Learn. Mem. Cognit. $25,702-720$.

Smith, M., and Wheeldon, L. (2004) Horizontal information flow in spoken sentence production. J. Exp. Psychol. Learn. Mem. Cognit. 30, 675-686.

Snyder, H. R., Hutchison, N., Nyhus, E., Curran, T., Banich, M. T., O’Reilly, R. C., and Munakata, Y. (2010). Neural inhibition enables selection during language processing. Proc. Natl. Acad. Sci. U.S.A. 107, 16483-16488.

Vigliocco, G., Vinson, D. P., and Siri, S. (2004). Semantic similarity and grammatical class in naming actions. Cognition 94, 91-100.

Wagner, V., Jescheniak, J. D., and Schriefers, H. (2010). On the flexibility of grammatical advance planning during sentence production: effects of cognitive load on multiple lexical access. J. Exp. Psychol. Learn. Mem. Cognit. 36, 423-440.

Conflict of Interest Statement: The authors declare that the research was conducted in the absence of any commercial or financial relationships that could be construed as a potential conflict of interest.

Received: 18 January 2011; accepted: 15 April 2011; published online: 02 May 2011.

Citation: Janssen $N$ and Caramazza A (2011) Lexical selection in multi-word production. Front. Psychology 2:81. doi: 10.3389/fpsyg.2011.00081

This article was submitted to Frontiers in Language Sciences, a specialty of Frontiers in Psychology.

Copyright (C) 2011 Janssen and Caramazza. This is an open-access article subject to a non-exclusive license between the author and Frontiers Media SA, which permits use, distribution and reproduction in other forums, provided the original authors and source are credited and other Frontiers conditions are complied with. 


\section{APPENDIX}

Table A1 | Nouns of low and high frequency ranges used in the experiment.

\begin{tabular}{|c|c|c|c|}
\hline LF range & LF range & HF range & HF range \\
\hline ant & lizard & apple & heart \\
\hline axe & moose & car & horse \\
\hline carrot & mouse & chair & jacket \\
\hline clown & nut & church & key \\
\hline comb & owl & cigar & knife \\
\hline couch & scarf & cloud & ladder \\
\hline cradle & sled & coat & lamp \\
\hline dart & snail & corn & lion \\
\hline dice & sock & crown & nose \\
\hline drill & stool & desk & safe \\
\hline drum & swan & dog & sheep \\
\hline eagle & toe & door & star \\
\hline fern & turtle & dress & table \\
\hline flute & vase & eye & thumb \\
\hline frog & vest & finger & top \\
\hline funnel & wagon & fish & train \\
\hline hanger & whale & flag & tree \\
\hline harp & worm & flower & truck \\
\hline kite & wrench & foot & turkey \\
\hline ladle & zipper & hair & window \\
\hline
\end{tabular}

LF, low frequency; HF, high frequency. 